\title{
EPCs-Collagen Sponge Complex Promotes Neovascularization of Chronic Cerebral Ischemia following Multiple Burr Hole (MBH) Surgery
}

Dongwei Dai ${ }^{1}$, Xiaoxi Zhang ${ }^{1}$, Qiong Lu ${ }^{2}$, Qiangjun $\mathbf{W u}^{3}$, Qinghai Huang ${ }^{1}$, Bo Hong ${ }^{1}$, Qiang Li ${ }^{1}$, Zifu Li ${ }^{1}$, Yina Wu ${ }^{1}$, Wenyuan Zhao ${ }^{1^{*}}$ and Jianmin Liu ${ }^{1^{*}}$

${ }^{1}$ Department of Neurosurgery, Changhai Hospital, Second Military Medical University, Shanghai, PR China

${ }^{2}$ Department of Laboratory Medicine, Changhai Hospital, Second Military Medical University, Shanghai, PR China

${ }^{3}$ Department of Neurosurgery, Jinhua Center Hospital, Zhejiang, China

"Corresponding author: Wenyuan Zhao, Department of Neurosurgery, Changhai Hospital, Second Military Medical University, 168 Changhai Road, Shanghai 200433, PR China, E-mail: chstroke@126.com

Jianmin Liu, Department of Neurosurgery, Changhai Hospital, Second Military Medical University, 168 Changhai Road, Shanghai 200433, China, Tel: +86-21-3116-1784;Fax: +86-21-3116-1784; E-mail: chstroke@163.com

Rec date: Jun 04, 2016; Acc date: Jul 06, 2016; Pub date: Jul 08, 2016

Copyright: ( 2016 Dai P, et al. This is an open-access article distributed under the terms of the Creative Commons Attribution License, which permits unrestricted use, distribution, and reproduction in any medium, provided the original author and source are credited.

\begin{abstract}
Objective: The aim of this study was to explore the effect of endothelial progenitor cells (EPCs)-collagen sponge complex on the neovascularization of chronic cerebral ischemia following multiple burr hole (MBH) surgery.

Methods: Bone marrow-derived EPCs from 5 Fisher 344 (F344) inbred rats were cultured and amplified in vitro, then co-cultured to become the EPCs-collagen sponge complex. Chronic cerebral hypo-perfusion was induced in 30 inbred male F344 rats by permanent bilateral internal carotid artery occlusion (BICAO). The MBH operation was performed three days after BICAO surgery. Rats were then randomly divided into 3 groups ( $n=10$ each group); EPCs-collagen sponge complex $+\mathrm{MBH}$; collagen sponge $+\mathrm{MBH}$ and $\mathrm{MBH}$ group. Either EPCs-collagen sponge complex or collagen sponge was implanted at the burr hole site in the EPCs-collagen sponge complex $+\mathrm{MBH}$ and the collagen sponge $+\mathrm{MBH}$ group, respectively. Regional cerebral blood flow (rCBF) was measured by Laser Doppler Flowmetry (LDF), EPCs were tracked by fluorescein, and immune-histochemical analysis for the neovascularization was performed 21 days after MBH surgery.
\end{abstract}

Results: Before the MBH surgery, no differences of rCBF between the EPCs-collagen sponge complex $+\mathrm{MBH}$ group, the collagen sponge $+\mathrm{MBH}$ group and the $\mathrm{MBH}$ alone group $(\mathrm{P}>0.05)$ were found. The increase of rCBF in the EPCs-collagen sponge complex $+\mathrm{MBH}$ group $(20.29 \pm 10.21 \%)$ was significantly higher when compared with the other two groups 21 days after MBH surgery $(7.39 \pm 6.85 \%$ and $10.32 \pm 6.27 \%$, respectively; $\mathrm{P}<0.05)$. The amount of new blood vessels in the EPCs-collagen sponge complex $+\mathrm{MBH}$ group was also significantly greater than the other two groups $(78.2 \pm 4.7$ vs. $48.7 \pm 6.1$ vs. $46.9 \pm 7.3 ; P<0.05)$. The labeled EPCs were incorporated into the endothelial cells of vessels at the burr hole sites.

Conclusion: The EPCs-collagen sponge complex enhances angiogenesis after $\mathrm{MBH}$ surgery under chronic cerebral hypo-perfusion condition, and increases the blood flow perfusion in ischemic cerebral tissue.

Keywords: Angiogenesis; Endothelial progenitor cells; Collagen; Multiple burr hole $(\mathrm{MBH})$ surge

\section{Introduction}

Chronic cerebral ischemia often leads to cerebral infarction and requires bypass surgery. Multiple burr hole surgery $(\mathrm{MBH})$, characterized as indirect bypass surgery, is relatively simple and safe and can create trans-galeal and/or transdural anastomoses from external arteries to an ischemic brain area via angiogenesis. This surgery has been demonstrated to be effective in the management of cerebral ischemia caused by moyamoya disease in children [1].

The degree of neo-anastomoses is a significant evaluation index for the effectiveness of the operation. However, suboptimal collateral vessel formation has been found in some adult moyamoya patients, as well as the unfavorable effect of stenosis or occlusion of cerebral arteries caused by atherosclerosis [1-3].
Therefore, successful promotion of postoperative neovascularization requires urgent attention. Several studies [4-6] have reported that endothelial progenitor cells (EPCs) promote the neovascularization of collateral vessels at the ischemic areas, thus further improving tissue perfusion. Collagen sponge is widely known as an excellent stereocarrier for cell growth [7-9]. Whether EPCs-collagen sponge complex enhances angiogenesis following $\mathrm{MBH}$ surgery under chronic cerebral hypo-perfusion condition was investigated in this study, and whether this complex can further increase ischemic cerebral perfusion was also explored.

\section{Materials and Methods}

\section{Animals and grouping}

Bone marrow from 5 Fisher 344 (F344) male inbred rats (250-300 g) was cultured and amplified in vitro. The remaining 30 F344 male inbred rats (250-300 g) were randomly divided into three groups (10 in 
Page 2 of 6

each group): EPCs-collagen sponge complex $+\mathrm{MBH}$ group, collagen sponge $+\mathrm{MBH}$ group and $\mathrm{MBH}$ alone group. All rats were anesthetized with $10 \%$ chloral hydrate via abdominal cavity injection, and were kept warm at the temperature of $37^{\circ} \mathrm{C}$ with heating pad when performing $\mathrm{MBH}$ surgery or bilateral internal carotid arteries ligation operation. Rats were provided by the Laboratory Animal Center affiliated to Shanghai Second Military Medical University. All animal protocols were approved by the Shanghai Second Military Medical University Animal Care Committee.

\section{Construction of the EPCs-collagen sponge complex}

\section{Isolation and in vitro culture of EPCs}

Animals were sacrificed through cervical dislocation after induction of general anesthesia with $10 \%$ chloral hydrate via abdominal cavity injection. Bone marrow cells were collected from the rat bone marrow by flushing the marrow cavity of bilateral femora with phosphate buffered saline (PBS). Mononuclear cells were separated by density gradient centrifugation $\left(2000 \mathrm{r} / \mathrm{min}, 37^{\circ} \mathrm{C}\right)$ using lymphocyte isolation, centrifuged with PBS twice, added into EGM-2 MV medium (Lonza, USA), and plated on to fibronectin coated $25 \mathrm{~mm}^{2}$ culture flasks. Thereafter, the cells were incubated $\left(37^{\circ} \mathrm{C}, 5 \% \mathrm{CO}_{2}\right) .3$ days later, nonadherent cells were removed along with the PBS, and fresh EGM-2 MV medium was added to the adherent cells. The morphology analysis of cultured cells was evaluated through phase contrast microscopy.

\section{Cellular staining}

Fluorescent detection of the adherent EPCs was performed at day 7 since culture. Direct fluorescent staining was conducted to detect dual binding of TITC-labeled Ulex europaeus agglutinin-1 (TITC-UEA-1; Sigma) and 1,1'-dioctadecyl-3,3,3,3'-tetramethylindocarbocyanine (DiI)-labeled acetylated low density lipoprotein (DiI-ac-LDL; Invitrogen). Cells were first incubated with DiI-ac-LDL (12 ug/mL) for $4 \mathrm{~h}$ at $37^{\circ} \mathrm{C}$ and then fixed with $1 \%$ paraformaldehyde for $10 \mathrm{~min}$. After washing 3 times with PBS, cells were incubated with TITC-UEA-1 (10 $\mathrm{ug} / \mathrm{mL}$ ) for $1 \mathrm{~h}$. The samples were observed with an inverted fluorescent microscope (Leica, Wetzlar, Germany). Cells stained with red, green and double-positive fluorescence were identified as DiI-acLDL positive cells, TITC-UEA-1 positive cells and differentiating EPCs, respectively.

\section{Construction of EPCs-collagen sponge complex}

Before the construction of the EPCs-collagen sponge complex, dual binding of CM-DiI and Hoechst 33342 was performed for the study of EPC tracking. Prior to cellular transplantation, cells were first washed with PBS 3 times and incubated with Hoechst $33342(10 \mathrm{ug} / \mathrm{mL})$ in PBS for $15 \mathrm{~min}$, and then incubated with CM-DiI $(2.5 \mathrm{ug} / \mathrm{mL})$ in PBS at $37^{\circ} \mathrm{C}$ for $5 \mathrm{~min}$ and $4^{\circ} \mathrm{C}$ for $15 \mathrm{~min}$. All samples were observed with an inverted fluorescent microscope (Leica, Germany). Cell membrane stained with red fluorescence and blue fluorescence was identified as CM-DiI positive cells and Hoechst 33342 positive cells, respectively.

Cells were then detached from the culture flask using Trypsin, washed with PBS and centrifuged. Following cell counting by hemocytometer, $1 \times 106$ cells were placed on top of a sterile gelfoam sponge $(5 \mathrm{~mm} \times 5 \mathrm{~mm}$ ). The media was supplemented with EGM-2 MV (Lonza, USA), and cells were sub-cultured in 6-well plates at $37^{\circ} \mathrm{C}$ under $5 \% \mathrm{CO}_{2}$ atmospheric conditions.

\section{Chronic cerebral hypo-perfusion model}

Bilateral internal carotid arteries (ICAs) were exposed via a midline cervical incision, sparing the sympathetic and vagal nerves. Ligation of bilateral ICAs was performed with 4-0 silk sutures.

\section{MBH surgery and implantation of the EPCs-collagen sponge complex or collagen sponge}

At day 3 after the permanent bilateral internal carotid artery occlusion (BICAO), all rats were fixed in prone position with a stereotactic head holder. The scalp was then incised at the parietooccipital midline, the galea and periosteum peeled off the skull, and the galeal flap prepared. A $6 \mathrm{~mm}^{2}$ craniectomy was performed by drilling just behind the coronal suture, $1 \mathrm{~mm}$ lateral from the midline, and dural incision was performed with microsurgical forceps and a microscope for protection of brain tissue. EPCs-collagen sponge complex or collagen sponge was then placed into bone defects.

\section{Measurement of rCBF}

The rCBF was measured 4 times: before, immediately after BICAO, before encephalo galeo synangiosis (EGS) and 21 days after EGS. The rCBF was measured with a transcranial laser Doppler flowmeter (LDF) (Moor, UK). Rats were anesthetized with halothane and fixed in a stereotactic head holder. A midline scalp skin incision was made, and the flowmeter probe was fixed. rCBF was measured and calculated in each rat at the bilateral frontal lobe and the cerebellum. The calculated value percentage of each frontal $\mathrm{rCBF}$ was compared with the ipsilateral rCBF of the cerebellum.

\section{Histology and immunohistochemistry}

Rats were deeply anesthetized 21 days after BICAO, and their aortas perfused with $4 \%$ paraformaldehyde in PBS. Brain tissue (along with EGS tissue) was removed, fixed with $4 \%$ paraformaldehyde for $6 \mathrm{~h}$, dehydrated with sucrose and embedded in optimum cutting temperature compound. Coronal sections measuring $5 \mathrm{~mm}$ and including EGS tissue were obtained from each brain.

The sections were sequentially incubated with antibody against von Willebrand factor (vWF; 1: 200), and stained with hematoxylin and eosin (H\&E) for histological evaluation. Sections were photographed at 40x magnification. Five photographs from different sites in the cerebral cortex were obtained from each brain section $(n=10)$. The amount and diameter of vWF-positive vessels in each section were recorded. Sections were analyzed blindly by 2 independent co-authors.

\section{Data analysis}

Data are expressed as mean \pm standard error (SE). Alterations in laser Doppler flowmetry (LDF) were calculated as percentage of measured blood flow/ipsilateral cerebellar blood flow. Student's t-test was performed and statistical significance was considered as less than 0.05 .

\section{Results}

Characteristics of EPCs cultured in vitro and the construction of EPCs-collagen sponge complex After 48 hours in vitro culture on fibronectin coated flasks, adherent cells showed colony-like growth (Figure 1A). At day 7 of culture, the cells covered approximately $80 \%$ of 
Citation: Dai D, Zhang X, Lu Q, Wu Q, Huang Q, et al. (2016) EPCs-Collagen Sponge Complex Promotes Neovascularization of Chronic Cerebral Ischemia following Multiple Burr Hole (MBH) Surgery. J Neurol Disord 4: 274. doi:10.4172/2329-6895.1000274

Page 3 of 6

the flask area. The cultured cells had the function of phagocytizing acLDL and binding UEA-1, the same with endothelial cells.
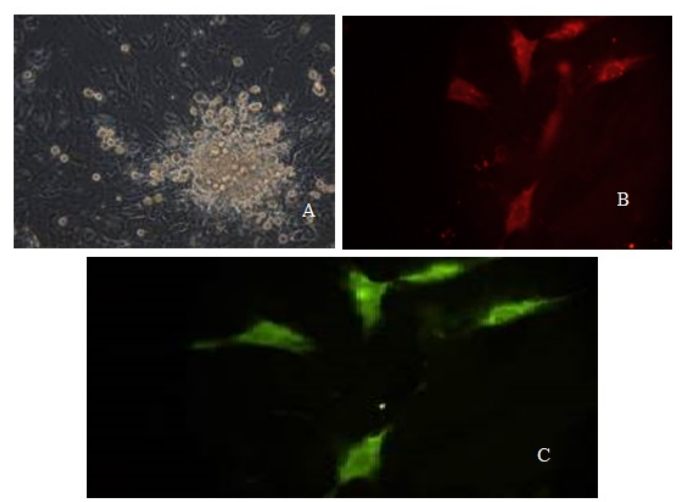

Figure 1: Adherent cells showed colony-like growth (A). Endocytosis of acLDL (red fluorescence) (B) and binding to UEA (green fluorescence) (C) identified EPCs. All images are shown at 40x magnification.

Plating $1 \times 106$ EPCs on to the collagen sponge in an in vitro coculture model. At day 7, small amount of cells attached to the collagen sponge. However, at day 14 greater amounts of cells attached to the collagen sponge, grew and formed the complex.

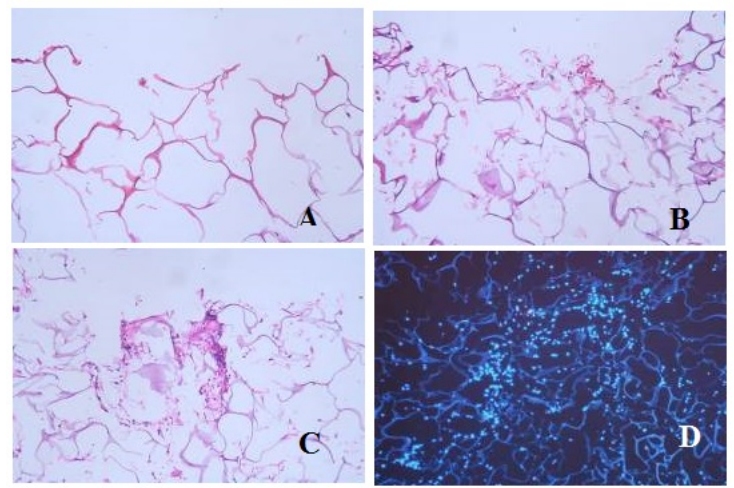

Figure 2: Collagen sponge has a good biocompatibility with EPCs, which is a successful in vitro three dimensional scaffold for EPCs culture. $H \& E$ staining of blank collagen sponge (A). H\&E staining of EPCs-collagen sponge complex at day 7, few cells attached to the collagen sponge and amplified (B). H\&E staining at day 14, many cells grew and attached to the collagen sponge (C). Nucleus stained blue was identified as Hoechst 33342 labeled positive cells at day 14 (D). All images are shown at $40 \times$ magnification.

\section{Changes of rCBF}

No significance of the changes of $\mathrm{rCBF}$ between three groups before and after BICAO and pre-MBH surgery $(\mathrm{P}>0.05)$ was observed. 21 days after $\mathrm{MBH}$ surgery, the $\mathrm{rCBF} \%$ of the EPCs-collagen sponge complex $+\mathrm{MBH}$ group was significantly higher $(74.80 \pm 7.85 \%)$ than that of the other two groups (collagen sponge $+\mathrm{MBH}$ group, $64.07 \pm$
$7.05 \%$ and $\mathrm{MBH}$ alone group, $61.63 \pm 10.12 \%, \mathrm{P}<0.05$ ) (Figure 2 and $3)$. The increase in the $\mathrm{rCBF} \%$ of the EPCs-collagen sponge complex + MBH group $(20.29 \pm 10.21 \%)$ was higher than that of the other two groups (collagen sponge $+\mathrm{MBH}$ group, $7.39 \pm 6.85 \%$ and $\mathrm{MBH}$ alone group, $10.32 \pm 6.27 \%, \mathrm{P}<0.05$ ) (Figure 4 and 5 ).

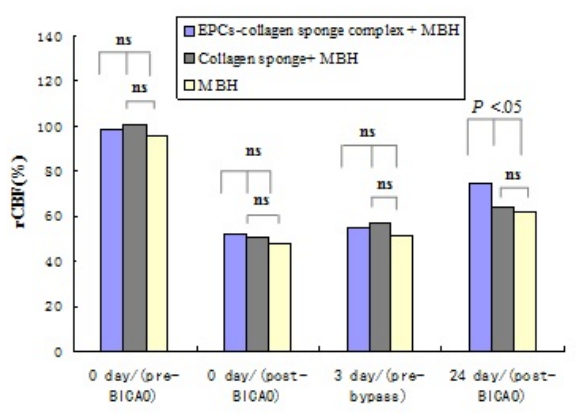

Figure 3: The rCBF in three groups before and after BICAO, preand post-MBH. The $\mathrm{rCBF} \%$ of the EPCs-collagen sponge complex + $\mathrm{MBH}$ group was significantly higher than the other two groups 21 days after MBH $(\mathrm{P}<0.05)$. Data are presented as mean \pm SE. ns: not significant.

\section{Histological assessment of fluorescence-labeled EPCs}

Both Hoechst 33342 and CM-DiI fluorescence labeling was used to identify the EPCs of the collagen sponge complex to confirm that the EPCs had reconstructed new vessels at the burr hole sites. Dualfluorescently labeled EPCs were detected from tissue located at areas between the brain and galea at day 21 after MBH surgery (Figure 6).

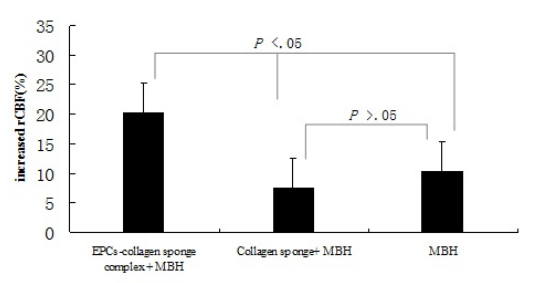

Figure 4: The $\mathrm{rCBF} \% 21$ days after $\mathrm{MBH}$ surgery was increased in each group. The increase in $\mathrm{rCBF} \%$ in the EPCs-collagen sponge complex $+\mathrm{MBH}$ group was significantly higher than the other two groups. Data are presented as mean $\pm \mathrm{SE}$.

\section{Immunohistochemistry}

Immunohistochemistry staining for von Willebrand factor (vWF) protein expression, a marker of angiogenesis, was performed in each of the experimental groups at day 21 after MBH surgery. A boundary zone between the cerebrum and the galea tissue was identified. vWF protein positive vessels staining was observed visually with a microscope under $40 \mathrm{x}$ magnification. The EPCs-collagen sponge complex $+\mathrm{MBH}$ group had significantly more new vessels 
(angiographic score: $78.2 \pm 4.7$ vs. $48.7 \pm 6.1$ vs. $46.9 \pm 7.3, \mathrm{P}<0.05$ ) and more intense positive staining for $\mathrm{vWF}$ protein.

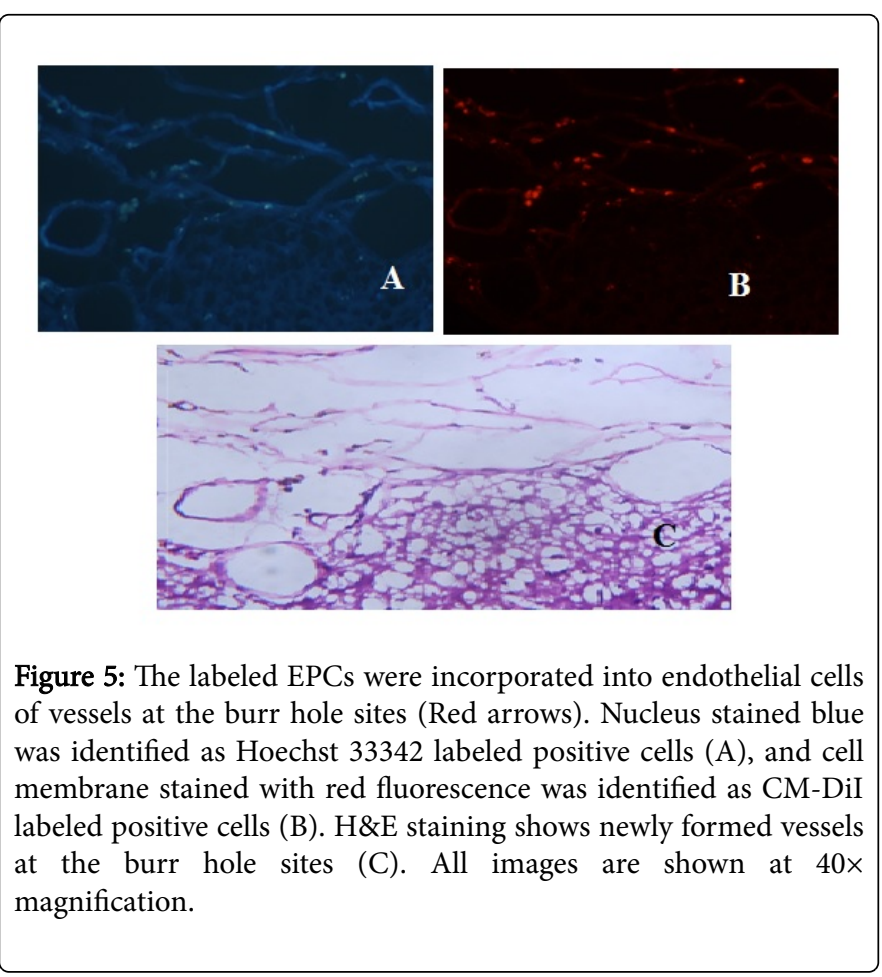

\section{Discussion}

The rat BICAO model utilized in this study effectively simulated human chronic cerebral hypo-perfusion conditions of moyamoya and atherosclerosis as previously reported [10,11]. In this study, $\mathrm{MBH}$ surgery and indirect revascularization were performed on the 3rd day after BICAO to better simulate human cerebral hypo-perfusion disease. Furthermore, preservation of the blood flow of the external carotid artery was beneficial in fundamental research of the neovascularization of chronic cerebral ischemia following $\mathrm{MBH}$ surgery. Ohta et al. [10] showed that this model simulates human cerebral hypo-perfusion disease better at day 3 after BICAO.

Interpretation of this study is as follows: Firstly, EPCs-collagen sponge- complex and collagen sponge are biocompatible, as indicated in this study. A satisfactory complex by co-culture in vitro was also obtained. Secondly, EPCs, the precursor cells of neo-endovascular cells, promote the neovascularization of the collateral vessels at ischemic brain areas. And finally, EPCs-collagen sponge complex promote neo-anastomoses from external arteries to an ischemic brain via angiogenesis under hypo-perfusion condition after $\mathrm{MBH}$ surgery, then further increase the ischemic cerebral perfusion.

The in vitro spindle-shaped cells, commonly known as EPCs, were first discovered and named by Asahara et al. [12]. In this study, the EPC cells expressed endothelial cell antigen and formed cord-like structures. EPCs transplantation, as previous studies reported, successfully improve capillary density and promote neovascularization of ischemic tissues $[5,13,14]$. The effects of EPCs-collagen sponge complex in the neovascularization of the chronic cerebral ischemia remain to be reported.
The results of the current study indicate that the EPCs-collagen sponge complex promotes neovascularization of the collateral vessels from the external carotid system to ischemic brain tissue following $\mathrm{MBH}$ surgery. As precursor cells, EPCs play an important role in neoangiogenesis and vasculogenesis [12], particularly in the neovascularization and remodeling of collateral vessels $[15,16]$.

Unsatisfactory quality and quantity of EPCs in moyamoya or atherosclerosis [17], diabetes and incremental age [18] are key factors influencing the efficiency of neovascularization. In vitro transplantation of EPCs has proved to be helpful in solving the problem of EPC deficiency, providing a sufficient reserve of EP cells for neovascularization. Previous studies have reported that collagen sponges are satisfactory three-dimensional fibrin matrices $[9,19,20]$. Bilic et al. [19] used 3D collagen I matrices and embedded preterm amnion epithelial to create a cell-matrix system that mimicked the structure of native amnion, which could also be used in the basic research of bone and skin tissue engineering $[9,20]$.

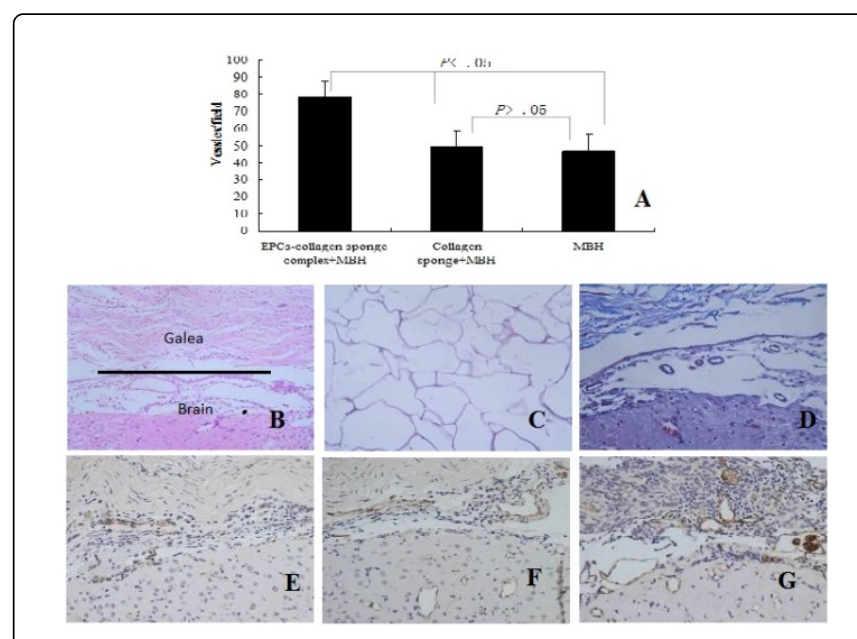

Figure 6: Vessels were located at the boundary zone between the cerebrum and the galea tissue in each group 21 days after $\mathrm{MBH}$ surgery. The EPCs-collagen sponge complex $+\mathrm{MBH}$ group had more new vessels than the other two groups $(\mathrm{P}<0.05)(\mathrm{A}) . \mathrm{H} \& \mathrm{E}$ staining (B), Masson staining (D) of the boundary zone between the cerebrum (lower side) and the galea tissue (upper side). Masson staining (C) for blank collagen sponge in vitro. Masson staining of the residual collagen sponge in the EPCs-collagen sponge complex $+\mathrm{MBH}$ group (the red arrow, (D) 21 days after $\mathrm{MBH}$ surgery. Immunohistochemical staining for $\mathrm{vWF}$ in sections from 3 different animals in each group: (E), $\mathrm{MBH}$ alone group; $(\mathrm{F})$, the collagen sponge + $\mathrm{MBH}$ group; $(\mathrm{G})$, the EPCs-collagen sponge complex + $\mathrm{MBH}$ group. All images are shown at $40 \times$ magnification.

Collagen sponges containing the Arg-Gly-Asp (RGD) sequence induce cell adhesion, cellular proliferation and differentiation, thus promoting neo-angiogenesis [21,22]. The advantages of this collagen sponge complex as scaffolds in $\mathrm{MBH}$ surgery include: 1) collagen sponges induce cell adhesion and cell proliferation inside pores, and prevent cell loss as seen by the increased flow of CSF; 2) this collagen sponge complex performed as a bridge grafting between dural lining and brain tissue as collagen sponges placed into the dural incision significantly increased EPCs proliferation, thereby stimulating angiogenesis; 3 ) the complex transplantation at bone defects positions EPCs at ischemic areas, thus avoiding bone marrow mobilization and 
homing, and increases initial seeding density and 4) the results of the study show that the in vitro co-culture model has excellent biocompatibility, and that at day 14 this in vitro modification produces a capillary vascular bed. Consequently, this study shows that for tissue engineering purposes, the in vitro co-culture model, which combines EPCs and collagen sponges along with the transplantation of EPCs and capillary vascular beds in bone defects, promotes neo-angiogenesis, otherwise known as the cellular-level vascular bypass.

Antioxidant, a molecule that inhibits the oxidation of other molecules, has been reported for therapeutic use in several disorders [1]. However, clinical trials of pharmacological neuro-protective effect in stroke were disappointing. Till now, two endogenous mechanisms have been identified, namely ischemic pre-conditioning and ischemic post-conditioning. The neuro-protective concept of preconditioning is based on the fact that a transient, non-injurious episode of ischemia will protect the brain from a subsequent longer ischemic shock. Postconditioning, a modified reperfusion subsequent to a prolonged ischemic episode, may also confer ischemic neuro-protection [23].

It is commonly accepted that MBH surgery and indirect revascularization is a simple, safe and generally non-invasive procedure of brain, with no risk of hyper-perfusion syndrome and limitation of vessel diameter and location, and is suitable for pediatric patients suffering from Moyamoya disease [1,24]. Previous studies have reported that, $\mathrm{MBH}$ surgery is an effective procedure in childhood cases of Moyamoya disease, which will decrease the recurrence rate of ischemic events following surgery $[1,25]$.

However, the therapeutic effect is unsatisfactory for cerebral hypoperfusion resulting from major cerebral vessel stenosis or occlusion in adult Moyamoya disease or atherosclerosis [1,3]. Indeed, several studies have speculated that EPCs deficiency and/or dysfunction is associated with inactive angiogenesis [12]. In coordination with those results, EPCs-collagen sponge complex $+\mathrm{MBH}$ therapy might promote neovascularization following $\mathrm{MBH}$ surgery in adult Moyamoya or atherosclerosis-relevant cerebral hypo-perfusion.

\section{Conclusion}

EPCs-collagen sponge complex enhances neo-angiogenesis at the burr hole site in a rat model of cerebral hypo-perfusion. Furthermore, the EPCs-collagen sponge complex increases blood flow perfusion in rat ischemic cerebral tissue.

\section{Acknowledgement}

This paper was financially supported by National Science Foundation of China (Project No. 81502163,81271271).

\section{References}

1. Alonso-Alconada D, Alvarez A, Arteaga O (2013) Neuroprotective effect of melatonin: a novel therapy against perinatal hypoxia-ischemia. International journal of molecular sciences 14: 9379-9395.

2. Mizoi K, Kayama T, Yoshimoto T, Nagamine Y (1996) Indirect revascularization for moyamoya disease: is there a beneficial effect for adult patients. Surg Neurol 45: 541-548.

3. Komotar RJ, Starke RM, Otten ML (2009) The role of indirect extracranial-intracranial bypass in the treatment of symptomatic intracranial atheroocclusive disease. J Neurosurg 110: 896-904.

4. Fan Y, Shen F, Frenzel T, Zhu W, Ye J, et al. (2010) Endothelial progenitor cell transplantation improves long-term stroke outcome in mice. Ann Neurol 67: 488-497.
5. Kamei N, Kwon SM, Kawamoto A, Ii M, Ishikawa M, et al. (2012) Contribution of bone marrow-derived endothelial progenitor cells to neovascularization and astrogliosis following spinal cord injury. J Neurosci Res 90: 2281-2292.

6. Hosseinkhani H, Hong PD, Yu DS, Chen YR, Ickowicz D, et al. (2012) Development of $3 \mathrm{D}$ in vitro platform technology to engineer mesenchymal stem cells. Int J Nanomedicine 7: 3035-3043.

7. Critser PJ, Voytik-Harbin SL, Yoder MC (2011) Isolating and defining cells to engineer human blood vessels. Cell Prolif 44 Suppl 1: 15-21.

8. Rodrigues CV, Serricella P, Linhares AB (2003) Characterization of a bovine collagen-hydroxyapatite composite scaffold for bone tissue engineering. Biomaterials 24: 4987-4997.

9. Ohta H, Nishikawa H, Kimura H, Anayama H, Miyamoto M (1997) Chronic cerebral hypoperfusion by permanent internal carotid ligation produces learning impairment without brain damage in rats. Neuroscience 79: 1039-1050.

10. Ohmori Y, Morioka M, Kaku Y (2011) Granulocyte colony-stimulating factor enhances the angiogenetic effect of indirect bypass surgery for chronic cerebral hypoperfusion in a rat model. Neurosurgery 68: 1372-1379.

11. Asahara T, Murohara T, Sullivan A (1997) Isolation of putative progenitor endothelial cells for angiogenesis. Science (New York, NY) 275: 964-967.

12. Qiu J, Li W, Feng S (2013) Transplantation of bone marrow-derived endothelial progenitor cells attenuates cerebral ischemia and reperfusion injury by inhibiting neuronal apoptosis, oxidative stress and nuclear factor-kappaB expression. International journal of molecular medicine 31: 91-98.

13. Cheng Y, Guo S, Liu G (2012) Transplantation of bone marrow-derived endothelial progenitor cells attenuates myocardial interstitial fibrosis and cardiac dysfunction in streptozotocin-induced diabetic rats. International journal of molecular medicine 30: 870-876.

14. Ghani U, Shuaib A, Salam A, Nasir A, Shuaib U, et al. (2005) Endothelial progenitor cells during cerebrovascular disease. Stroke 36: 151-153.

15. Asahara T, Masuda H, Takahashi T (1999) Bone marrow origin of endothelial progenitor cells responsible for postnatal vasculogenesis in physiological and pathological neovascularization. Circulation research 85: 221-228.

16. Kim JH, Jung JH, Phi JH (2010) Decreased level and defective function of circulating endothelial progenitor cells in children with moyamoya disease. Journal of neuroscience research 88: 510-518.

17. Waltenberger J (2001) Impaired collateral vessel development in diabetes: potential cellular mechanisms and therapeutic implications. Cardiovascular research 49: 554-560.

18. Bilic G, Hall H, Bittermann AG (2005) Human preterm amnion cells cultured in 3-dimensional collagen I and fibrin matrices for tissue engineering purposes. American journal of obstetrics and gynecology 193: 1724-1732.

19. Mafi P, Hindocha S, Mafi R (2012) Evaluation of biological protein-based collagen scaffolds in cartilage and musculoskeletal tissue engineering-a systematic review of the literature. Current stem cell research \& therapy. 7: 302-309.

20. Kidd KR, Nagle RB, Williams SK (2002) Angiogenesis and neovascularization associated with extracellular matrix-modified porous implants. Journal of biomedical materials research 59: 366-377.

21. Stadlinger B, Pilling E, Mai R (2008) Effect of biological implant surface coatings on bone formation, applying collagen, proteoglycans, glycosaminoglycans and growth factors. Journal of materials science Materials in medicine 19: 1043-1049.

22. Pignataro G, Scorziello A, Di Renzo G, Annunziato L (2009) Postischemic brain damage: effect of ischemic preconditioning and postconditioning and identification of potential candidates for stroke therapy. FEBS J 276: 46-57.

23. Kawaguchi T, Fujita S, Hosoda K, Shose Y, Hamano S, et al. (1996) Multiple burr-hole operation for adult moyamoya disease. J Neurosurg 84: 468-476. 
Citation: Dai D, Zhang X, Lu Q, Wu Q, Huang Q, et al. (2016) EPCs-Collagen Sponge Complex Promotes Neovascularization of Chronic Cerebral Ischemia following Multiple Burr Hole (MBH) Surgery. J Neurol Disord 4: 274. doi:10.4172/2329-6895.1000274

Page 6 of 6

24. Oliveira RS, Amato MC, Simão GN, Abud DG, Avidago EB, et al. (2009) Effect of multiple cranial burr hole surgery on prevention of recurrent ischemic attacks in children with moyamoya disease. Neuropediatrics 40: 260-264.
25. Hill JM, Zalos G, Halcox JP, Schenke WH, Waclawiw MA, et al. (2003) Circulating endothelial progenitor cells, vascular function, and cardiovascular risk. N Engl J Med 348: 593-600. 